\title{
Planejamento da logística de suprimento de plataformas Offshore por meio de um modelo matemático 2L-CVRP com frota heterogênea e equilíbrio náutico
}

\author{
Bianca Passos Arpini ${ }^{1}$ e Rodrigo de Alvarenga Rosa ${ }^{2}$
}

\begin{abstract}
Resumo: No Brasil a exploração de petróleo ocorre, sobretudo, no mar onde são implantadas plataformas offshore que estão distantes da costa. Logo, é fundamental planejar a logística de suprimento por meio de navios. Este artigo propõe um modelo matemático de Programação Linear Inteira Mista denominado Weight Balance Two-Dimensional Loading Heterogeneous Fleet Vehicle Routing Problem (WB2L-HFVRP) aplicado ao planejamento da logística de suprimento de plataformas offshore visando a criação de rotas que considerem o equilíbrio náutico e a melhor arrumação das cargas no convés. Este modelo se diferencia por considerar frota heterogênea e utilizar uma função objetivo que visa minimizar o número de navios, a distância navegada e a diferença entre os pesos distribuídos entre os bordos do navio para manter o equilíbrio náutico. Testou-se o modelo em instâncias baseadas em dados reais da Petrobras no CPLEX 12.6 e alcançou-se uma redução de até $25 \%$ em relação à distância real navegada.

Palavras-chave: 2L-CVRP, WB2L-HFVRP, Logística de Suprimento, Plataformas Offshore.
\end{abstract}

\begin{abstract}
Oil is vital in the world, and in Brazil the exploration occurs mainly on the sea where offshore platforms are implanted far from the coast. Therefore it is important to plan the logistics of supply through offshore support vessels. This article proposes a mathematical model of Mixed Integer Linear Programming named Weight Balance Two-Dimensional Loading Heterogeneous Fleet Vehicle Routing Problem (WB2L-HFVRP) applied to the offshore platform supply logistics planning aiming to create routes that consider the nautical balance and the better stowage of cargo on deck. This model differs from other models because it considers heterogeneous fleet and uses a objective function that aims to minimize the number of ships, the sailed distance, and the difference between the weights distributed between the sides of the ship, the nautical balance. The model was tested on instances based on real data from Petrobras in CPLEX 12.6 and achieved a reduction of at most $25 \%$ compared to the actual traveled distance.
\end{abstract}

Keywords: 2L-CVRP, WB2L-HFVRP, Supply Logistics, Offshore platforms.

\section{INTRODUÇÃO}

O petróleo é atualmente a principal fonte energética do mundo, sendo importante para vários setores econômicos, sobretudo o de transportes e o petroquímico. A exploração deste recurso no Brasil se fundamenta principalmente na exploração em mar onde são implantadas plataformas offshore que possuem uma demanda constante por suprimentos e são abastecidas por uma frota de navios que partem de portos denominados bases de apoio. No pré-sal os campos de produção podem estar situados a mais de $300,0 \mathrm{~km}$ da costa brasileira (Ferro e Teixeira, 2009). Isso torna a operação de suprimento mais complexa, pois as maiores distâncias aumentarão o tempo médio de viagem dos navios entre o porto e as unidades offshore. Dessa forma, um planejamento eficiente da logística para suprimento de material é vital para a exploração do pré-sal.

Os principais navios utilizados para este transporte são os navios denominados Platform Supply Vessel (PSV), que só podem transportar as cargas no nível do convés não podendo empilhá-las por questões de segurança de navegação. As cargas não podem ser rotacionadas em função de

\footnotetext{
1 Bianca Passos Arpini, Programa de Pós Graduação em Engenharia Civi Universidade Federal do Espírito Santo. (bianca.p.arpini@gmail.com)

2 Rodrigo de Alvarenga Rosa, Programa de Pós Graduação em Engenharia Civil, Universidade Federal do Espírito Santo.

(rodrigo.a.rosa@ufes.br)

Manuscrito recebido em 18/05/2015 e aprovado para publicação em 21/09/2015.

Este artigo é parte de TRANSPORTES v. 23, n. 4, 2015. ISSN: 2237-1346
} (online). DOI:10.14295/transportes.v23i4.920 suas dimensões e são carregadas e descarregadas por guindastes. Existe um tempo limite máximo de três dias que o PSV pode ficar em viagem sem regressar ao porto.

Visando o planejamento da logística de suprimento de plataformas, este artigo propõe um modelo de Programação Linear Inteira Mista (PLIM) que é denominado Weight Balance Two-Dimensional Loading Heterogeneous Fleet Vehicle Routing Problem (WB2L-HFVRP), baseado no Capacitated Vehicle Routing Problem with Two-dimensional Loading Constraints (2L-CVRP), que elabora para cada navio uma rota e sua arrumação da carga no convés para atender a várias plataformas. Sua função objetivo visa à minimização do número de navios utilizados, da distância navegada, e da diferença entre os pesos distribuídos entre os bordos do navio com o intuito de manter o equilíbrio náutico. A principal diferença do modelo proposto em relação aos modelos existentes na literatura reside no fato de o modelo incorporar frota heterogênea e tratar do equilíbrio náutico do navio.

Ressalta-se que, apesar de existirem algumas publicações acerca do planejamento de navios que incluem, sobretudo, modelos periódicos que visam à determinação da composição da frota e horários semanais dos navios (Fagerholt e Lindstad, 2000; Halvorsen-Weare e Fagerholt, 2011; Panamarenka, 2011; Halvorsen-Weare et al., 2012; Shyshou et al., 2012; Norlund e Gribkovskaia, 2013); e modelos não periódicos, que incluem aspectos como a determinação de rotas, composição e dimensionamento da frota ótima e capacidade de armazenagem da instalação offshore (Brejon e Brinati, 1998; Aas et al., 2007; Gribkovskaia et al., 2008; Ribeiro e Iachan, 2009; Almeida, 2009; Lopes, 
2011; Vianna et al., 2012; Henty et al.,2012; Vaquero et al., 2012 ; Friedberg e Uglane, 2013; Sopot e Gribkovskaia, 2014), nenhuma delas trabalhou de forma integrada a roteirização com a arrumação da carga no navio usando o 2LCVRP, tampouco o equilíbrio náutico.

O artigo é organizado como segue: na Seção 2 temse uma revisão com trabalhos relevantes sobre o 2L-CVRP. $\mathrm{Na}$ Seção 3 apresenta-se o modelo matemático proposto. $\mathrm{Na}$ Seção 4 são apresentados os experimentos computacionais. $\mathrm{Na}$ Seção 5 têm-se a apresentação e a análise dos resultados e na Seção 6 têm-se as conclusões.

\section{PROBLEMA DE ROTEIRIZAÇÃO DE VEÍCULOS COM RESTRIÇÕES DE CARREGAMENTO EM DUAS DIMENSÕES}

O 2L-CVRP é uma combinação de dois problemas NP-hard: o CVRP e o Two-Dimensional Bin Packing Problem (2BPP) (Leung et al., 2011). No 2LCVRP, os clientes demandam a entrega de um conjunto de itens retangulares, que serão transportados por veículos com uma dada capacidade de peso e uma superfície de carregamento bidimensional. Tais itens possuem tamanhos e pesos determinados e não podem ser empilhados. Todos os itens de um dado cliente devem ser atribuídos a um único veículo. Todos os veículos, por sua vez, devem começar e terminar no depósito central e os itens transportados devem possuir uma arrumação viável dentro da superfície de carregamento desses veículos.

O objetivo do problema é colocar e arrumar os itens dentro dos veículos e entregá-los aos clientes por meio de uma rede de transportes com mínimo custo total, ou seja, redução da distância percorrida e o número de veículos utilizados (Iori et al., 2007; Fuellerer et al., 2009; Leung et al., 2010).

O 2L-CVRP visa atribuir os clientes $i$ a uma das rotas, tal que o custo de transporte total seja minimizado, e que para cada rota exista um carregamento viável de itens dentro da superfície de carregamento do veículo. O 2L-CVRP pode ser classificado como sequencial e irrestrito. No carregamento sequencial a disposição da carga no veículo deve obedecer à ordem inversa de descarga, análogo a um sistema LIFO (Last In - First Out) (Gendrau et al., 2008; Zachariadis et al., 2009; Fuellerer et al., 2009; Araujo, 2010; Leung et al., 2011). No irrestrito a disposição dos itens não inclui a restrição de sequenciamento e em geral, este caso corresponde a veículos que podem ser descarregados verticalmente com um guindaste (KhebbacheHadji et al., 2013). Quanto ao carregamento, o 2L-CVRP também pode ser orientado ou não orientado. No orientado os itens possuem uma orientação fixa, não podendo ser rotacionados. No não orientado os itens podem ser rotacionados em $90^{\circ}$ (Fuellerer et al., 2009).

Iori et al. (2007) introduziram o 2L-CVRP propondo um modelo de Programação Linear Inteira (PLI) e o uso de Branch-and-Cut (B\&C) e Branch-and-Bound (B\&B). Gendreau et al, (2008) propuseram o uso de Tabu Search (TS). Fuellerer et al. (2009) empregaram Ant Colony Optimization (ACO) para o 2L-CVRP com carregamento não orientado. Zachariadis et al. (2009) propuseram a Guided Tabu Search (GTS). Azevedo et al. (2009) utilizaram B\&C para o 2L-CVRP. Strodl et al. (2010) e Wei et al. (2015) utilizaram Variable Neighborhood Search (VNS). Leung et al. (2010) apresentaram Simulated Annealing (SA). Leung et al. (2011) propuseram Extended Guided Tabu Search (EGTS). Duhamel et al. (2011) propuseram Greedy Randomized Adaptive Search Procedure (GRASP) combinada com Evolutionary Local Search (ELS). Shen e Murata (2012) usaram Genetic Algorithm (GA). Zachariadis et al. (2013) propuseram Promise Routing-Memory Packing (PRMP). Bin et al. (2013) apresentaram Artificial Bee Colony Algorithm (ABC). Dominguez et al. (2014a) apresentaram um modelo de PLI e utilizaram Multistart Biased Randomized Algorithm (MS-BR). AbdalHammed et al. (2014) propuseram a meta-heurística Large Neighborhood Search (LNS).

Entre as variantes do 2L-CVRP, Malapert et al. (2008) apresentaram o Two-Dimensional Pickup and Delivery Routing Problem with Loading Constraints (2L-PDP). Muñoz (2011) apresentou uma modelagem para o 2L-CVRP denominada Capacitated Vehicle Routing Problem with Two-dimensional Loading Constraints and Handling Costs (2L-CVRP-H), cuja função objetivo minimiza os custos de roteirização e os custos de manipular os itens no local de demanda. Hamdi-Dhaoui et al. (2012) propuseram a Meta-heurística Nondominated Sorting Genetic Algorithm II (NSGA - II) para resolver o problema Two-dimensional Loading Capacitated Vehicle Routing Problem with Partial Conflicts (2LPC-CVRP) com função biobjetivo onde se considerou a minimização o custo de transporte bem como o equilíbrio da carga. Leung et al. (2013) abordaram o Two-Dimensional Loading Heterogeneous Fleet Vehicle Routing Problem (2L-HFVRP) e usaram a Meta-heurística Simulated Annealing with Heuristic Local Search (SA-HLS). Khebbache-Hadji et al. (2013) trataram o two-dimensional Loading Capacitated Vehicle Routing Problem with Time Windows (2L-CVRPTW) usando a Meta-heurística Memetic Algorithm (MA). Côté et al. (2013) abordaram o Vehicle Routing with Stochastic TwoDimensional Items (S2L-CVRP) e utilizaram o método L-shaped inteiro. Martínez e Amaya (2013) apresentaram o Vehicle Routing Problem With Multi-Trips, Time Windows And Two-Dimensional Circular Loading Constraints (VRPM-TW-CL) e utilizaram Tabu Search (TS). Dominguez et al. (2014b) resolveram o 2L-HFVRP com rotação dos itens para as versões irrestritas do problema, utilizando o MS-BR.

Depois desta revisão, não se encontrou nenhum artigo que tratasse do $2 \mathrm{~L}-\mathrm{CVRP}$ com as características de frota heterogênea e equilíbrio náutico, ou seja, o equilíbrio de peso entre os dois bordos do navio, que são as características principais do WB2L-HFVRP proposto neste artigo.

\section{MODELO MATEMÁTICO PROPOSTO}

O modelo proposto trata de um problema de roteirização com frota heterogênea de navios com carregamento bidimensional e equilíbrio de peso entre os bordos do navio, que foi denominado por este artigo como sendo Weight Balance Two-Dimensional Loading Heterogeneous Fleet Vehicle Routing Problem (WB2L-HFVRP), que consiste em uma nova contribuição para o estudo desse problema, já 


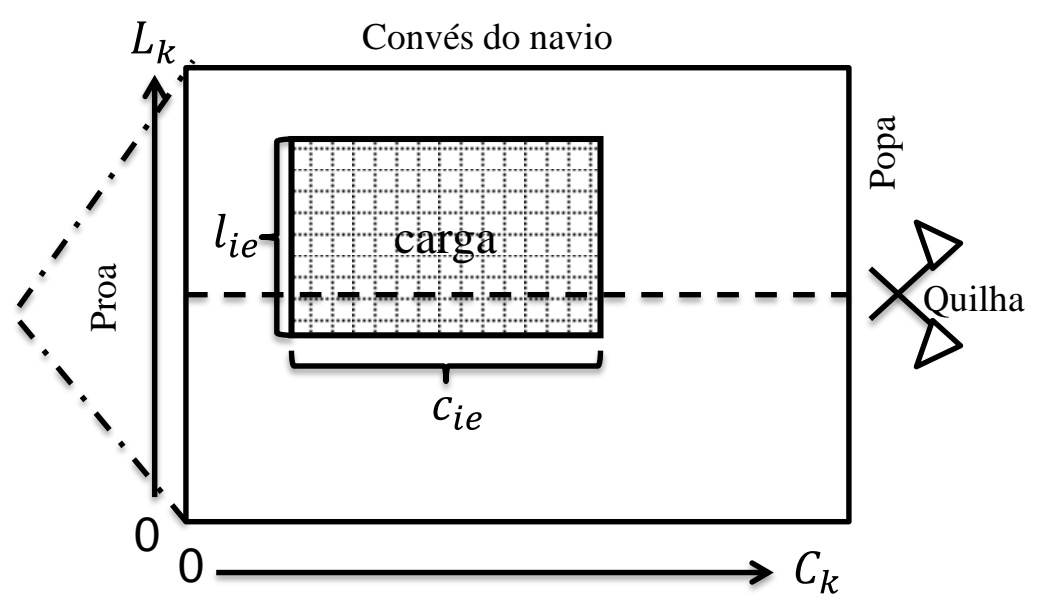

Figura 1. Representação esquemática da superfície de carregamento no navio

que, até então, nenhum trabalho incorporou frota heterogênea e equilíbrio náutico do navio em um mesmo modelo.

O WB2L-HFVRP visa criar para cada navio uma rota e sua respectiva arrumação da carga no convés para atender a várias plataformas. As cargas não podem ser empilhadas, sendo posicionadas no piso do convés, portanto, é uma arrumação bidimensional. Cada plataforma pode ser atendida por um único navio, para o qual existe um tempo limite máximo de viagem. O problema é classificado como orientado e irrestrito do 2L-CVRP, pois as cargas não poderão ser rotacionadas e serão retiradas dos navios pela parte superior via guindastes, sendo desnecessárias restrições de sequenciamento.

Define-se o WB2L-HFVRP como um grafo não orientado completo $G=(N T, A), \quad$ em que $N T=\{0,1, \ldots, n p+1\}$ é o conjunto de nós que correspondem ao porto $(n o ́ 0)$, às plataformas de petróleo (nós $1,2, \ldots, n p$ ), no qual $n p$ é o número de plataformas, e ao nó virtual $(n p+1)$, e $A=\left\{a_{i j} \mid i, j \in N\right\}$ é o conjunto de arcos, que liga uma plataforma $i$ a uma plataforma $j$. Seja $N$ o conjunto de plataformas $(1, \ldots, n p), N 0=\{0\} \cup N$ e $N 1=N \cup\{n p+1\} ; K$ o conjunto de navios disponíveis no porto $(1, \ldots, k)$, em que $k$ é o número de navios da frota; $P^{i}$ conjunto de cargas demandadas por uma plataforma $i \in N$. A cada navio $k \in K$ são associados os parâmetros largura $L_{k}$ do convés de carga em metros (m); comprimento $C_{k}$ do convés de carga em metros (m); capacidade máxima $Q_{k}$ do convés de carga em toneladas (t); custo fixo $C F_{k}$ em reais $(\mathrm{R} \$)$; custo variável $C V_{k}$ em reais por $\mathrm{Km}$ (R\$/Km); e tempo limite de operação $T L_{k}$ em horas (h). A cada carga $e \in P^{i}$ estão relacionados os parâmetros largura $l_{i e}$ da carga $e$ solicitada pela plataforma $i$ em metros (m); comprimento $c_{i e}$ da carga $e$ solicitada pela plataforma $i$ em metros (m); e peso $q_{i e}$ da carga $e$ solicitada pela plataforma $i$ em toneladas (t), todos definidos em $i \in N, e \in P_{i}$. A pla- taforma $i \in N T$ tem como parâmetros a distância em quilômetros entre ela e as outras plataformas, $d_{i j}\left(d_{i i}=0\right)$, o tempo em horas para percorrer a distância $d_{i j}, t_{i j}$, e o tempo de atendimento a um navio, $t a_{i}$. Para restrições com ativação utilizou-se o parâmetro $M$, que denota um valor para lógica muito grande. Para conferir maior peso à parcela referente ao momento na função objetivo, usou-se o parâmetro de calibração $\mu$.

No WB2L-HFVRP, considera-se um diagrama comprimento-largura, equivalente ao primeiro quadrante do plano cartesiano, em que o eixo horizontal e o eixo vertical representam o comprimento e a largura do convés de um navio, respectivamente, e o canto inferior esquerdo corresponde à coordenada $(0,0)$. As cargas devem ser analisadas em um plano bidimensional, ou seja, são consideradas áreas retangulares $\left(U_{e}=c_{i e} l_{i e}\right)$. A Figura 1 mostra uma representação do convés do navio contendo uma carga, demonstrando suas respectivas dimensões e posicionamento em relação ao primeiro quadrante do plano cartesiano. Destacase o eixo da quilha do navio na Figura 1, pois é em relação a este eixo, que deverá ser calculado o equilíbrio náutico.

No WB2L-HFVRP, um carregamento viável deve satisfazer as seguintes restrições: 1) todas as cargas de uma plataforma devem ser transportadas por um mesmo navio e entregas fracionadas não são permitidas; 2) todos os itens devem ter orientação fixa, ou seja, não é possível rotacionálos, e devem ser carregados com seus lados paralelos aos lados da superfície de carregamento; 3 ) cada navio começa e termina sua viagem no porto; 4) cada plataforma deve ser visitada apenas uma única vez; 5) a capacidade, comprimento e largura de cada navio não devem ser excedidos; 6) dois itens não podem ser sobrepostos em um navio.

No que se refere às variáveis de decisão, há três categorias: 1) variáveis de carregamento, 2) variáveis de roteirização e 3) variáveis relativas ao peso.

Em relação à primeira categoria, há duas classes: 1) as variáveis indicando a posição das cargas e 2) variáveis indicando a posição relativa das áreas retangulares das cargas no diagrama comprimento-largura. No que tange ao primeiro grupo, têm-se três variáveis inteiras positivas definidas em $k \in K, i \in N, e \in P^{i}: \alpha_{k i e}$, que designa a posição inicial de colocação da carga $e$ da plataforma $i$ em relação ao 


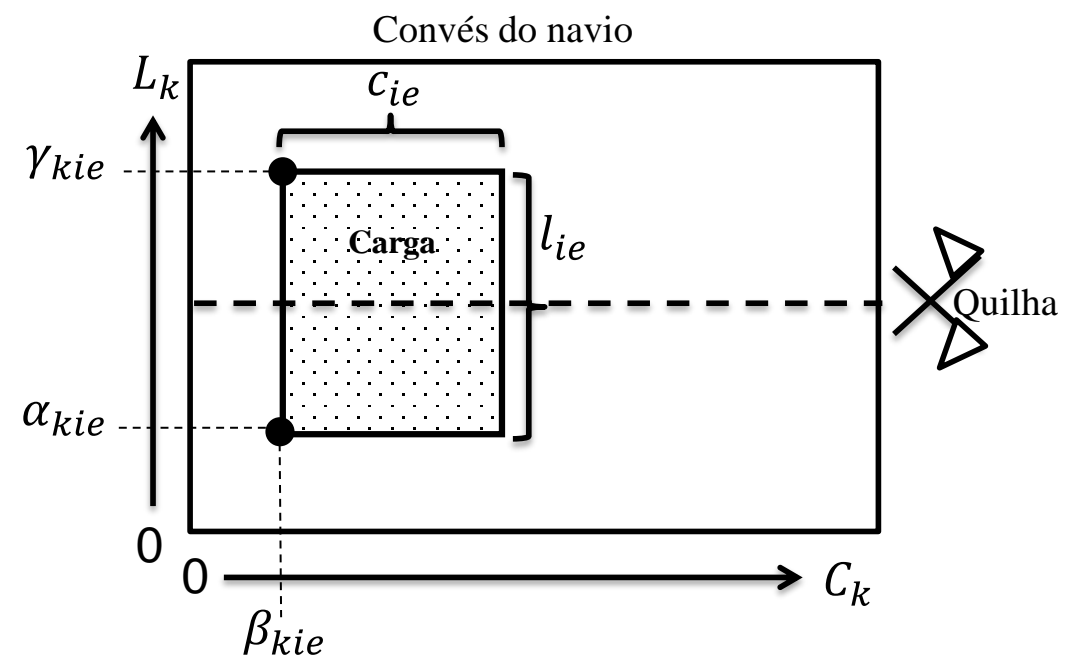

Figura 2. Representação gráfica das variáveis de posição das cargas

eixo da largura $(y)$ do navio $k ; \beta_{\text {kie }}$ que indica a posição inicial de colocação da carga $e$ da plataforma $i$ em relação ao eixo de comprimento $(x)$ do navio $k$; e $\gamma_{k i e}$ que determina a posição final de colocação da carga $e$ da plataforma $i$ em relação ao eixo da largura $(y)$ do navio $k$. Para exemplificar, a Figura 2 ilustra as três variáveis de posição em relação ao convés do navio citadas anteriormente.

No que diz respeito às variáveis indicando a posição relativa das áreas retangulares das cargas no diagrama comprimento-largura, há duas variáveis binárias: $\sigma_{\text {kieif }}$, que assume valor 1 , se a área retangular da carga $e$ está completamente abaixo da área retangular da carga $f$ e as duas áreas retangulares não estão sobrepostas, $\forall k \in K, i, j \in N, e \in P^{i}, f \in P^{j}$, e 0 caso contrário; e $\delta_{k i e j f}$, que assume valor 1 , se a área retangular da carga $e$ está completamente à esquerda da área retangular da carga $f$ e as duas áreas retangulares não estão sobrepostas, $\forall k \in K, i, j \in N, e \in P^{i}, f \in P^{j}$, e 0 caso contrário.

No que concerne às variáveis do VRP, têm-se duas variáveis binárias: $x_{k i j}$, que assume valor 1 , se o navio $k$ viaja diretamente da plataforma $i$ para a plataforma $j$, $\forall k \in K, i, j \in N T$, e 0 caso contrário; e $y_{k i}$, que assume valor 1 , se o navio $k$ atende a plataforma $i$, $\forall k \in K, i \in N 0$, e 0 caso contrário.

Por fim, há as variáveis relativas ao peso. Nesta categoria há duas classes: a) variável para equilíbrio de peso e b) variável para indicar peso transportado. A variável referente ao equilíbrio de peso no navio é denotada por $w_{k} \mathrm{e}$ designa o momento do navio $k, k \in K$. A variável para indicar peso transportado é denotada por $q t_{k i}$ e indica o peso transportado pelo navio $k \in K$ quando ele chega à plataforma $i \in N$.

Com base nos conjuntos, parâmetros e variáveis de decisão apresentadas anteriormente, a seguir são apresentadas a função objetivo e as restrições do modelo matemático proposto.

$$
\begin{aligned}
& \text { Função Objetivo } \\
& \operatorname{Min} \sum_{k \in K} \sum_{i \in N T} \sum_{j \in N T} C V_{k} d_{i j} x_{k i j}+\sum_{k \in K} \sum_{j \in N} C F_{k} x_{k 0 j}+\sum_{k \in K} \sum_{i \in N} \sum_{e \in P^{i}} \beta_{k i e}+\mu \sum_{k \in K} w_{k} \\
& \text { Restrições } \\
& y_{k i}=\sum_{j \in N 1} x_{k i j} \\
& \alpha_{k j f}-\alpha_{k i e}-l_{i e}-\left(\left(\sigma_{\text {kieif }}-1\right) L_{k}\right)+\left(\left(2-y_{k i}-y_{k j}\right) M\right) \geq 0 \\
& \forall k \in K, \forall i \in N 0, i \neq j \\
& \beta_{k j f}-\beta_{k i e}-c_{i e}-\left(\left(\sigma_{k i e j f}-1\right) C_{k}\right)+\left(\left(2-y_{k i}-y_{k j}\right) M\right) \geq 0 \\
& \forall k \in K, \forall i, j \in N, \forall e \in P^{i}, \forall f \in P^{j}, i \neq j \\
& \left(\sigma_{\text {kiejf }}+\sigma_{\text {kjfie }}+\delta_{\text {kiejf }}+\delta_{k j f i e}\right)+\left(\left(2-y_{k i}-y_{k j}\right) M\right) \geq 1 \\
& \forall k \in K, \forall i, j \in N, \forall e \in P^{i}, \forall f \in P^{j}, i \neq j \\
& l_{i e} y_{k i}+\alpha_{k i e}=\gamma_{k i e} \\
& \forall k \in K, \forall i, j \in N, \forall e \in P^{i}, \forall f \in P^{j}, i \neq j \\
& \forall k \in K, \forall i \in N, \forall e \in P^{i} \\
& L_{k} \geq l_{i e} y_{k i} \\
& \forall k \in K, \forall i \in N, \forall e \in P^{i} \\
& \alpha_{k i e} \leq\left(L_{k}-l_{i e}\right) y_{k i}
\end{aligned}
$$


Planejamento da logística de suprimento de plataformas Offshore por meio de um modelo matemático 2L-CVRP com frota heterogênea e equilíbrio náutico

$$
\begin{aligned}
& C_{k} \geq c_{i e} y_{k i} \\
& \beta_{k i e} \leq\left(C_{k}-c_{i e}\right) y_{k i} \\
& \gamma_{k i e} \geq \alpha_{k i e} \\
& \sum_{i \in N} \sum_{e \in P^{i}} l_{i e} c_{i e} y_{k i} \leq L_{k} C_{k} \\
& \sum_{k \in K} \sum_{j \in N T} x_{k i j}=1 \\
& \sum_{j \in N} x_{k 0 j} \leq 1 \\
& \sum_{i \in N T} x_{k i h}-\sum_{j \in N T} x_{k h j}=0 \\
& \sum_{i \in N 0} x_{k i(n p+1)}=1 \\
& x_{k(n p+1) j}=0 \\
& x_{k i 0}=0 \\
& \sum_{i \in N} \sum_{e \in P^{i}} q_{i e} y_{k i} \leq Q_{k} \\
& \sum_{i \in N 0} \sum_{j \in N 1}\left(t_{i j}+t a_{i}\right) x_{k i j} \leq T L_{k} \\
& w_{k} \geq \sum_{i \in N} \sum_{e \in P^{i}}\left(\alpha_{k i e}-\left(\frac{\left(L_{k}-l_{k}\right)}{2}\right)\right) q_{i e} y_{k i} \\
& w_{k} \geq \sum_{i \in N} \sum_{e \in P^{i}}\left(\left(\frac{\left(L_{k}-l_{k}\right)}{2}\right)-\alpha_{k i e}\right) q_{i e} y_{k i} \\
& w_{k} \geq 0 \\
& q t_{k j} \geq q t_{k i}+\sum_{e \in P^{i}} q_{i e}-\left(M\left(1-x_{k i j}\right)\right) \\
& q t_{k i} \geq 0 \\
& q t_{k i} \leq Q_{k} \\
& \alpha_{\text {kie }} \in Z^{+} \\
& \beta_{\text {kie }} \in Z^{+} \\
& \gamma_{\text {kie }} \in Z^{+} \\
& w_{k} \in R^{+} \\
& q t_{k i} \in R^{+} \\
& x_{k i j} \in\{0,1\} \\
& y_{k i} \in\{0,1\} \\
& \delta_{\text {kiejf }} \in\{0,1\} \\
& \sigma_{\text {kiejf }} \in\{0,1\}
\end{aligned}
$$

A função objetivo, Equação (1), representa a minimização da distância navegada e da quantidade de navios usados, o que reduz os custos variáveis e fixos, respectivamente, do comprimento do convés do navio utilizado para a disposição das cargas e da diferença entre os pesos distribuídos entre os bordos do navio com o intuito de manter o equilíbrio náutico, sendo esta a contribuição mais importante do modelo proposto.

As Restrições (2) indicam se o navio $k$ vai para plataforma $i$ ou não. As Restrições (3) garantem que a largura

$$
\begin{aligned}
& \forall k \in K, \forall i \in N, \forall e \in P^{i} \\
& \forall k \in K, \forall i \in N, \forall e \in P^{i} \\
& \forall k \in K, \forall i \in N, \forall e \in P^{i} \\
& \forall k \in K, \forall i \in N, \forall e \in P^{i} \\
& \forall i \in N, i \neq j \\
& \forall k \in K \\
& \forall k \in K, \forall h \in N, i \neq h, j \neq h \\
& \forall k \in K \\
& \forall k \in K, \forall j \in N T \\
& \forall k \in K, \forall i \in N T \\
& \forall k \in K \\
& \forall k \in K, i \neq j \\
& \forall k \in K, L_{k} \geq l_{\text {ie }} \\
& \forall k \in K, L_{k} \geq l_{\text {ie }} \\
& \forall k \in K \\
& \forall k \in K, \forall i, j \in N, i \neq j \\
& \forall k \in K, \forall i \in N \\
& \forall k \in K, \forall i \in N \\
& \forall k \in K, \forall i \in N, \forall e \in P^{i} \\
& \forall k \in K, \forall i \in N, \forall e \in P^{i} \\
& \forall k \in K, \forall i \in N, \forall e \in P^{i} \\
& \forall k \in K \\
& \forall k \in K, \forall i \in N \\
& \forall k \in K, \forall i, j \in N T \\
& \forall k \in K, \forall i \in N 0 \\
& \forall k \in K, \forall i, j \in N, \forall e \in P^{i}, \forall f \in P^{j} \\
& \forall k \in K, \forall i, j \in N, \forall e \in P^{i}, \forall f \in P^{j}
\end{aligned}
$$

de uma carga não irá sobrepor a largura de outra carga. As Restrições (4) asseguram que o comprimento de uma carga não irá sobrepor o comprimento de outra carga. As Restrições (5) garantem que não haverá sobreposições das áreas retangulares das cargas e que a carga demandada por uma mesma plataforma vai ocupar apenas um dos navios. As Restrições (3), (4) e (5) têm em comum a parcela $\left(\left(2-y_{k i}-y_{k j}\right) M\right)$ que é utilizada para ativar as restrições. 
As Restrições (6) representam que a posição final de colocação da carga $e$ em relação ao eixo da largura $L_{k}$ do navio deve ser a soma da largura da carga, $l_{i e}$, com a posição de colocação inicial da carga $e$ em relação ao eixo da largura $L_{k}$ do navio.

As Restrições (7) garantem que a carga designada para o navio $k$ terá largura $l_{i e}$ menor ou igual que a largura $L_{k}$ do navio. As Restrições (8) asseguram que a posição de colocação inicial da carga $e$ em relação ao eixo da largura $L_{k}$ do navio deverá estar contida no intervalo de posicionamento que varia de 0 até a largura $L_{k}$ do navio subtraída da largura da carga, $l_{i e}$. Dessa forma, nenhuma carga será posicionada além dos limites de largura do convés do navio.

As Restrições (9) asseguram que a carga atribuída ao navio $k$ terá comprimento $c_{i e}$ menor ou igual que o comprimento $C_{k}$ do navio. As Restrições (10) garantem que a posição de colocação inicial de comprimento ocupada por cada carga $e$ em relação ao eixo do comprimento $C_{k}$ do navio deverá estar contida no intervalo de posicionamento que varia de 0 até o comprimento do navio $C_{k}$ subtraído do comprimento da carga $c_{i e}$. Assim, nenhuma carga será posicionada além dos limites de comprimento do convés do navio.

As Restrições (11) limitam os valores de $\gamma_{k i e}$, que devem ser obrigatoriamente maiores que o valor de $\alpha_{\text {kie }}$, que é a posição inicial de colocação da carga no eixo da largura do navio.

As Restrições (12) impõem que a área total ocupada pelos itens que estão em um dado navio deve ser menor ou igual à superfície de carregamento disponível no navio. As Restrições (13) garantem que cada plataforma $i$ seja servida por exatamente um navio. As Restrições (14), (15) e (16) representam restrições de fluxo em redes, que exigem que cada navio $k$ parta do porto (nó 0 ) somente uma vez, deixe o nó $h$ se e somente se entrar neste nó, e retorne ao porto $(n o ́ n p+1)$ somente uma vez.

As Restrições (17) impedem que o nó $n p+1$, que representa o porto ao final do fluxo, faça rota com outros nós. As Restrições (18) não permitem que os navios retornem ao nó 0 , que representa o porto no início do fluxo. As Restrições (19) impõem que a demanda total de cada rota do navio $k$ não excede sua capacidade $Q_{k}$. As Restrições (20) limitam o tempo máximo que o navio $k$ pode navegar.

As Restrições (21) e (22) conjugadas equivalem ao módulo de momento calculado para o navio. Para efetuar o cálculo do momento, considera-se somatória da diferença entre a metade da largura $L_{k}$ do convés e metade da largura $l_{i e}$ da carga, subtraída de $\alpha_{\text {kie }}$, e multiplicada pelo peso da carga. Essa somatória, minimizada na função objetivo, é multiplicada por um parâmetro de calibração $\mu=10$, pois ao adotar um peso maior para esta parcela, força-se que este valor seja o mais próximo de zero, de forma a garantir o equilíbrio. Já as Restrições (23) garantem a não-negatividade da variável $w_{k}$.

As Restrições (24) asseguram que o peso $q t_{k j}$ transportado pelo navio $k$ na plataforma $j$ seja maior ou igual ao peso $q t_{k i}$ transportado pelo navio $k$ quando chega à plataforma $i$ somado ao peso das cargas da plataforma $i$. A parcela $\left(M\left(1-x_{k i j}\right)\right)$ serve para ativar a restrição, ao verificar se o navio $k$ viaja direto da plataforma $i$ para a plataforma $j$. As Restrições (25) mostram que $q t_{k i}$ é maior ou igual a zero. As Restrições (26) garantem que o peso transportado pelo navio $k$ na plataforma $i$ não ultrapasse a capacidade $Q_{k}$ do navio. As Restrições (24) a (26) têm por função a eliminação de sub-rotas.

As Restrições (27), (28) e (29) indicam que $\alpha_{k i e}, \beta_{k i e}$ e $\gamma_{k i e}$ são variáveis inteiras positivas. As Restrições (30) e (31) mostram que $w_{k}$ e $q t_{k i}$ são variáveis reais positivas. As Restrições (32), (33), (34) e (35) indicam que $x_{k i j}, y_{k i}$, $\delta_{\text {kieif }}$ e $\sigma_{\text {kieif }}$ são variáveis binárias.

A formulação descrita anteriormente é não linear por causa das Restrições (21) e (22), já que para calcular o momento de cada navio ocorre a multiplicação da variável inteira $\alpha_{k i e}$ e da variável binária $y_{k i}$. Considerando que $0 \leq \alpha_{k i e} \leq\left(L_{k}-l_{i e}\right)$, sendo que $\left(L_{k}-l_{i e}\right)$ resulta em uma constante, tais restrições podem ser linearizadas por meio da introdução da variável $z_{k i e}$ e das Restrições (36) a (40).

$z_{k i e} \leq\left(L_{k}-l_{i e}\right) y_{k i} \forall k \in K, \forall i \in N, \forall e \in P^{i}, L_{k} \geq l_{i e}$

$z_{k i e} \leq \alpha_{k i e}$

$\forall k \in K, \forall i \in N, \forall e \in P^{i}$

$z_{k i e} \geq \alpha_{k i e}-\left(\left(L_{k}-l_{i e}\right)\left(1-y_{k i}\right)\right) \forall k \in K, \forall i \in N, \forall e \in P^{i}, L_{k} \geq l_{i e}$

$z_{\text {kie }} \geq 0$

$\forall k \in K, \forall i \in N, \forall e \in P^{i}$

$z_{\text {kie }} \in R^{+}$

$\forall k \in K, \forall i \in N, \forall e \in P^{i}$

Neste caso, se $y_{k i}=0$, tem-se que $z_{k i e}=0$. Se $y_{k i}=1$, então as Restrições (38) se tornam $z_{k i e} \geq \alpha_{k i e}$, que combinado com (37), implica $z_{k i e}=\alpha_{k i e}$. As Restrições (40) mostram que $z_{k i e}$ é variável real positiva. Assim, as Restrições (21) e (22) podem ser substituídas, respectivamente, pelas Restrições (41) e (42).

$$
\begin{array}{ll}
w_{k} \geq \sum_{i \in N} \sum_{e \in P^{i}}\left(z_{k i e}-\left(\frac{\left(L_{k}-l_{k}\right)}{2} y_{k i}\right)\right) q_{i e} & \forall k \in K, L_{k} \geq l_{i e} \\
w_{k} \geq \sum_{i \in N} \sum_{e \in P^{i}}\left(\left(\frac{\left(L_{k}-l_{k}\right)}{2} y_{k i}\right)-z_{k i e}\right) q_{i e} & \forall k \in K, L_{k} \geq l_{i e}
\end{array}
$$

\section{EXPERIMENTOS COMPUTACIONAIS}

Para efetuar os testes, criaram-se instâncias baseadas em dados reais da Petrobras, considerando a Bacia do Espírito Santo e a Bacia de Campos. 

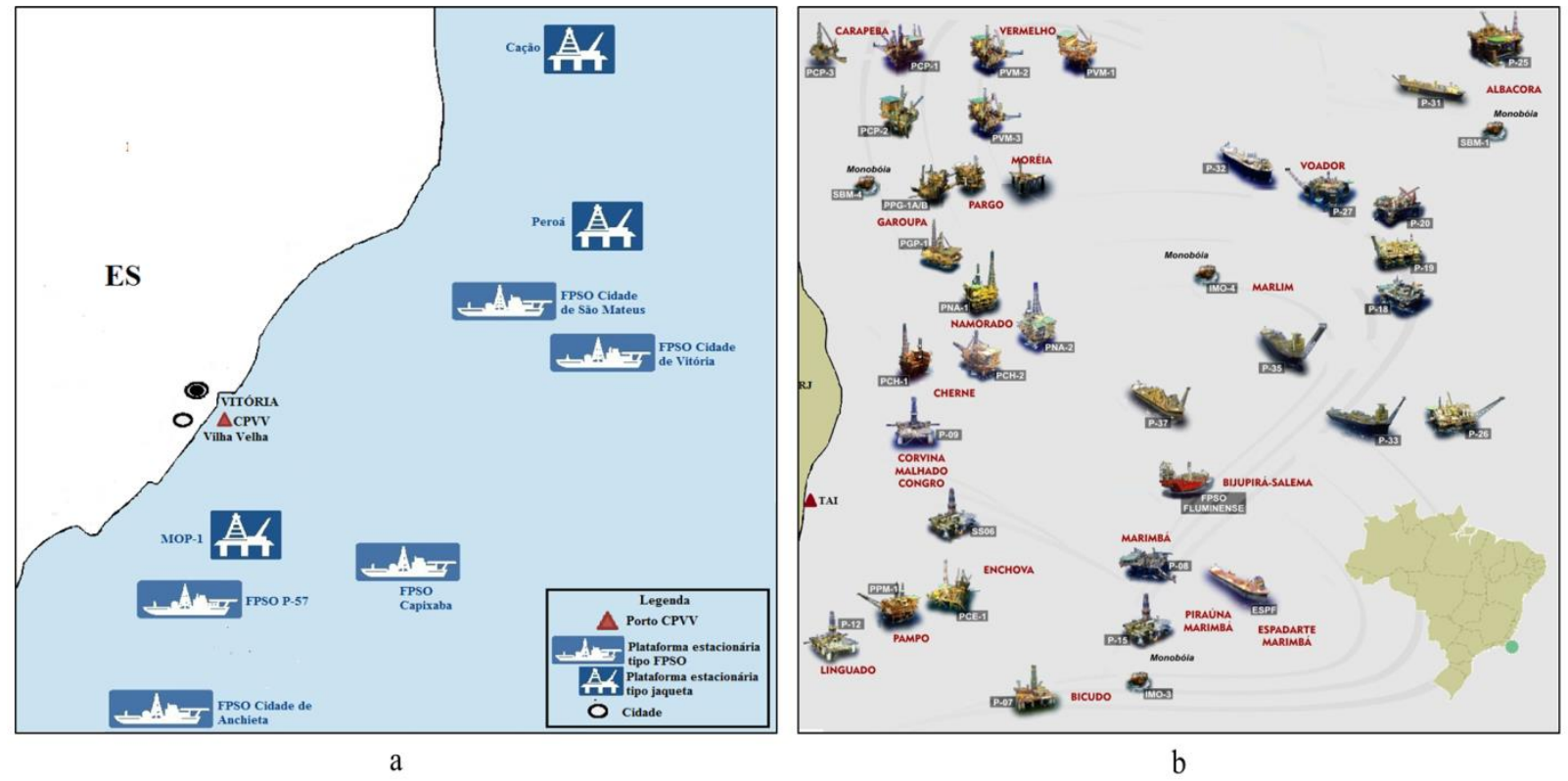

Figura 3. Localização das plataformas e dos portos: a) Bacia do ES; b) Bacia de Campos. Fonte: a) Adaptado da Página A Gazeta (2014); b) Adaptado da Página Click Macaé (2015).

O local de origem dos navios na Bacia do Espírito Santo é o porto Companhia Portuária de Vila Velha (CPVV), que é o maior porto de apoio a plataformas offshore do Espírito Santo. O local de origem na Bacia de Campos é o Terminal Alfandegado de Imbetiba (TAI), que é a maior base de apoio offshore no Brasil, situada em Macaé-RJ. Os locais de destino de ambas as bacias são as plataformas de produção. A Figura 3 ilustra o posicionamento das plataformas nas bacias estudadas bem como a posição dos respectivos portos.

A partir das latitudes e longitudes obtidas, calculouse a distância em linha reta das plataformas entre elas e em relação ao porto para cada Bacia, conforme Equação (43).

Distância $=6371 \cos ^{-1}\left(\begin{array}{l}\cos \left(\pi \frac{\left(90-\text { lat }_{j}\right)}{180}\right) \cos \left(\left(90-\text { lat }_{i}\right) \frac{\pi}{180}\right)+ \\ \sin \left(\left(90-\text { lat }_{j}\right) \frac{\pi}{180}\right) \sin \left(\left(90-\text { lat }_{i}\right) \frac{\pi}{180}\right) \cos \left(\left(\text { lon }_{i}-\text { lon }_{j}\right) \frac{\pi}{180}\right)\end{array}\right.$

Em que o valor 6371 é o raio da Terra em quilômetros; $l a t_{i}$ e $l a t_{j}$ são as latitudes das plataformas $i$ e $j$, respectivamente, em graus; e $l{ }_{0} n_{i}$ e $l o n_{j}$ são as longitudes das plataformas $i$ e $j$, respectivamente, em graus.

No que se refere aos navios, a Petrobras, em geral, utiliza três tipos de PSV: PSV 1500, PSV 3000 e PSV 4500, que possuem áreas de convés, custos fixos e variáveis diferentes. O navio pode realizar viagens de até três dias.

Criou-se um conjunto de instâncias em que $T L_{k}=72 h(\forall k)$ e outro que utiliza $T L_{k}=48 h(\forall k)$. O tempo médio para carregar o navio no porto é $t a_{0}=6 h$ e o tempo médio para atender às plataformas é $t a_{i}=2,5 h$, com $i=1, \ldots, n p$. A matriz de tempo foi obtida dividindo-se a matriz de distância pela velocidade média dos PSV $(18,52 \mathrm{Km} / \mathrm{h})$.

Foram criadas 17 instâncias que se diferenciam pela quantidade de plataformas, de dias ou de cargas totais. A nomenclatura usada é "PKDA $\mathrm{P}_{n} \mathrm{~B}$ ", em que P é a quantidade de plataformas; $\mathrm{K}$ é o número de navios disponibilizados; D é o prazo em dias limite da rota; $A_{n}$ é se a instância é real, " $R_{n}$ ", ou instância de teste " $T_{n}$ ", em que $n=1,2 \ldots, n$ e denota diferenças em instâncias com a mesma caraterística, por exemplo, número de cargas; e B indica a qual Bacia os dados fazem referência: Bacia do Espírito Santo, denotada por "E", ou Bacia de Campos, indicada por "C".

A principal diferença entre as instâncias do tipo " $\mathrm{R}$ " e " $T$ " é que as primeiras se baseiam em manifestos de cargas reais, e usam as demandas, dimensões e rotas reais, enquanto as de teste foram criadas para testar a capacidade de resolução do modelo, adotando-se, para isso, áreas e pesos de cargas próximos aos limites de capacidade dos navios de forma a forçar o uso de uma quantidade de navios maior que dois. Apenas as instâncias do tipo "R" permitem comparação com a situação real em termos de distância navegada. Ressalta-se que os dados das instâncias reais foram obtidos em manifestos de cargas da Petrobras de maio/2014 e de dezembro/2014.

A Tabela 1 apresenta a nomenclatura de cada instância; a quantidade de plataformas correspondente; a quantidade de PSVs disponíveis; a quantidade limite de dias que um navio pode navegar; a variação $(\Delta)$ de cargas demandadas por plataforma; e as cargas totais de cada instância. A coluna variação $(\Delta)$ de cargas demandadas por plataforma representa a variação possível, mínima e máxima, do número de cargas em cada uma das plataformas da instância testada.

Foi utilizado o solver CPLEX 12.6 para escrever o modelo proposto bem como para a execução de cada instância. Foi estabelecido um tempo limite para execução do modelo de 4 horas usando um computador Intel $i 7$ com 16 GB de memória. 
ARPINI, B.P.; ROSA, R.A.

Tabela 1. Conjunto de instâncias criadas

\begin{tabular}{|c|c|c|c|c|c|}
\hline Instância & $\begin{array}{l}\text { Quantidade de } \\
\text { Plataformas }\end{array}$ & $\begin{array}{l}\text { Quantidade de } \\
\text { PSV disponivel }\end{array}$ & $\begin{array}{l}\text { Quantidade } \\
\text { de dias }\end{array}$ & $\begin{array}{l}\Delta \text { de cargas por } \\
\text { plataforma }\end{array}$ & $\begin{array}{l}\text { Cargas } \\
\text { Totais } \\
\end{array}$ \\
\hline 432R__C & 4 & 3 & 2 & $1-6$ & 17 \\
\hline $432 \mathrm{R}_{2} \mathrm{C}$ & 4 & 3 & 2 & $1-8$ & 30 \\
\hline 532R $\mathrm{R}_{1 \_} \mathrm{C}$ & 5 & 3 & 2 & $1-4$ & 15 \\
\hline 533R $\mathrm{R}_{1 \_} \mathrm{C}$ & 5 & 3 & 3 & $1-4$ & 17 \\
\hline 632R $\mathrm{R}_{1 \_} \mathrm{C}$ & 6 & 3 & 2 & $1-4$ & 18 \\
\hline 732R $\mathrm{R}_{1 \_\mathrm{E}}$ & 7 & 3 & 2 & $1-3$ & 14 \\
\hline 733R 2_E & 7 & 3 & 3 & $1-3$ & 13 \\
\hline 833R1_E & 8 & 3 & 3 & $1-2$ & 13 \\
\hline 832T 1 1E & 8 & 3 & 2 & $1-2$ & 13 \\
\hline $832 \mathrm{~T}_{2} \_\mathrm{E}$ & 8 & 3 & 2 & $1-4$ & 29 \\
\hline $842 \mathrm{~T}_{1} \mathrm{E}$ & 8 & 4 & 2 & $1-4$ & 32 \\
\hline $842 \mathrm{R}_{1} \mathrm{C}$ & 8 & 4 & 2 & $1-8$ & 47 \\
\hline $933 R_{1} C$ & 9 & 3 & 3 & $1-1$ & 9 \\
\hline $1242 \mathrm{~T}_{1} \_\mathrm{C}$ & 12 & 4 & 2 & $1-2$ & 24 \\
\hline $1242 \mathrm{~T}_{2} \_\mathrm{C}$ & 12 & 4 & 2 & $1-4$ & 32 \\
\hline $1552 \mathrm{~T}_{1 \_\mathrm{C}}$ & 15 & 5 & 2 & $1-2$ & 30 \\
\hline $1552 \mathrm{~T}_{2} \_\mathrm{C}$ & 15 & 5 & 2 & $1-3$ & 45 \\
\hline
\end{tabular}

\section{APRESENTAÇÃO E ANÁLISE DE RESULTADOS}

Na Tabela 2 são apresentados os resultados obtidos pelos testes computacionais para cada instância, listando-se o número de navios que foram utilizados para atender as demandas das plataformas (1); a soma da distância, em Km, navegada por toda a frota usada (2); a soma dos momentos náuticos da frota usada (3); o GAP (4); e o tempo de execução em segundos (5). Também se mostram o número de navios (6) e a distância total percorrida (7) do caso real. Na coluna (8), avalia-se o ganho percentual obtido em termos de distância comparando-se o resultado do modelo com a situação real.

Ressalta-se que a função objetivo representa a minimização da somatória dos custos variáveis, diretamente relacionados com a distância percorrida; dos custos fixos, proporcionais à quantidade de navios usados; do comprimento do convés do navio utilizado e da diferença entre os pesos distribuídos entre os bordos do navio com o intuito de manter o equilíbrio náutico. Todas essas parcelas apresentam unidades dimensionais diferentes. Por isso, para facilitar a análise comparativa dos resultados, optou-se por mostrar na Tabela 2 especificamente, a quantidade de navios, a distância navegada e o momento total.

Analisando a Tabela 2, nota-se que o CPLEX conseguiu resolver otimamente oito das 17 instâncias, as quais estão destacadas em negrito na tabela.

No que tange ao total de instâncias criadas, 10 constituem cenários reais, sendo sete referentes à Bacia de Campos e três relativos à Bacia do Espírito Santo, representados pelas instâncias 432R $\mathrm{R}_{1} \mathrm{C}, 432 \mathrm{R}_{2} \mathrm{C}, 532 \mathrm{R}_{1} \mathrm{C}, 533 \mathrm{R}_{1 \_} \mathrm{C}$, 632R 1 _C, 732R $\mathrm{R}_{1} \mathrm{E}, \quad 733 \mathrm{R}_{2}$ E, 833R 1 E, 842R 1 C e 933R $\mathrm{R}_{1} \mathrm{C}$.

No que se refere à distância navegada das instâncias baseadas em dados reais, alcançou-se uma redução de $0,11 \%, 0,00 \%, 14,03 \%, 5,82 \%, 2,65 \%, 2,00 \%, 2,40 \%$,

Tabela 2. Resultados apresentados pelo CPLEX para as instâncias

\begin{tabular}{|c|c|c|c|c|c|c|c|c|}
\hline & \multicolumn{4}{|c|}{ WB2L-HFVRP (CPLEX) } & \multicolumn{3}{|c|}{ Real } & \multirow{2}{*}{$\begin{array}{c}\text { Ganho WB2L- } \\
\text { HFVRP } x \text { Real } \\
(\%) \\
(8) \\
\end{array}$} \\
\hline & $(1)$ & (2) & $(3)$ & (4) & $(5)$ & $(6)$ & $(7)$ & \\
\hline Instância & $\begin{array}{l}N^{o} \text { de } \\
\text { navios }\end{array}$ & $\begin{array}{l}\text { Distância } \\
\text { Total }(\mathrm{Km})\end{array}$ & $\begin{array}{l}w_{k} \text { Total } \\
(t . m)\end{array}$ & $G A P$ & $\begin{array}{l}\text { Tempo de } \\
\text { Execução }(s)\end{array}$ & $\begin{array}{l}N^{o} \text { de } \\
\text { navios }\end{array}$ & $\begin{array}{l}\text { Distância } \\
\text { Total }(\mathrm{km})\end{array}$ & $\begin{array}{c}\text { Distância Total } \\
(\%)\end{array}$ \\
\hline 432R1_C & 1 & 406,98 & 0,0085 & $0,04 \%$ & 14400 & 1 & 407,43 & $0,11 \%$ \\
\hline 432R $\mathrm{R}_{2} \mathrm{C}$ & 1 & 438,58 & 0,023 & $0,30 \%$ & 14400 & 1 & 438,58 & $0,00 \%$ \\
\hline 532R 1 _C & 1 & 305,70 & 0,103 & $0,04 \%$ & 14400 & 1 & 355,60 & $14,03 \%$ \\
\hline 533R 1 _C & 1 & 603,34 & 0,0085 & $0,07 \%$ & 14400 & 1 & 640,65 & $5,82 \%$ \\
\hline 632R 1 _C & 1 & 413,53 & 0,033 & $0,03 \%$ & 14400 & 1 & 424,78 & $2,65 \%$ \\
\hline 732R1_E & 2 & 548,91 & 0,0745 & $0,00 \%$ & 165,55 & 2 & 560,15 & $2,00 \%$ \\
\hline 733R2_E & 1 & 491,92 & 0,177 & $0,00 \%$ & 71,09 & 1 & 504,07 & $2,40 \%$ \\
\hline 833R1_E & 1 & 580,98 & 0,121 & $0,00 \%$ & 53,78 & 1 & 614,83 & $5,50 \%$ \\
\hline 832T1_E & 2 & 743,14 & 0,122 & $0,00 \%$ & 20,78 & - & - & - \\
\hline 832T $2 \_E$ & 3 & 1055,69 & 0,01 & $0,00 \%$ & 41,42 & - & - & - \\
\hline 842T 1 E & 4 & 1013,89 & 0,055 & $0,00 \%$ & 2,00 & - & - & - \\
\hline 842R1_C & 2 & 845,56 & 0,004 & $43,14 \%$ & 14400 & 2 & 846,01 & $0,05 \%$ \\
\hline 933R__C & 1 & 599,98 & 0,036 & $0,00 \%$ & 299,02 & 1 & 803,3 & $25,0 \%$ \\
\hline 1242T1_C & 4 & 1292,54 & 1,505 & $0,00 \%$ & 1185,97 & - & - & - \\
\hline 1242T2_C & 4 & 1357,56 & 0,055 & $0,01 \%$ & 14400 & - & - & - \\
\hline $1552 \mathrm{~T}_{1} \_\mathrm{C}$ & 5 & 1756,19 & 1,505 & $0,03 \%$ & 14400 & - & - & - \\
\hline $1552 \mathrm{~T}_{2} \_\mathrm{C}$ & 5 & 1756,19 & 0,895 & $23,17 \%$ & 14400 & - & - & - \\
\hline
\end{tabular}


Planejamento da logística de suprimento de plataformas Offshore por meio de um modelo matemático $2 \mathrm{~L}-\mathrm{CVRP}$ com frota heterogênea e equilíbrio náutico

$5,50 \%, 0,05 \%$ e $25,0 \%$ respectivamente em relação ao real. Isso representa uma economia de $357,25 \mathrm{Km}$, o que impactaria diretamente nos custos variáveis. Isso leva a concluir que caso fossem analisadas todas as operações desta empresa, poder-se-ia obter ganhos muito expressivos.

Quanto ao número de navios utilizados, não houve diferenças entre o real e o gerado pelo modelo, provavelmente, por conta das dimensões e pesos das cargas, o que não permitiria acomodar todas as cargas em um único navio.

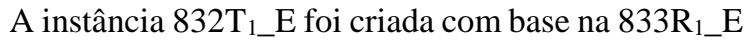
para avaliar a influência do tempo limite de viagem na formação das rotas para a Bacia do Espírito Santo. O tempo de $48 \mathrm{~h}$ foi fator determinante na configuração das rotas, uma vez que seria impossível, dadas as características de velocidade do navio e tempo adotadas, realizar uma rota envolvendo todas as plataformas no tempo de $48 \mathrm{~h}$. A partir disso, percebeu-se, para a Bacia do Espírito Santo, a tendência de clusterização de plataformas que se encontram ao norte do CPVV e das que se localizam ao sul do CPVV. Este tipo de agrupamento era esperado, dada à proximidade geográfica entre as plataformas posicionadas ao norte e ao sul do CPVV.

A solução da instância $832 \mathrm{~T}_{2}$ E, que contém 29 cargas, usou os três navios disponíveis e gerou três rotas com no máximo três plataformas. A instância $842 \mathrm{~T}_{1} \_$E, que tem 32 cargas ao todo e usou quatro navios, foi resolvida no menor tempo de execução entre as 17 instâncias, levando apenas dois segundos. Isso se deve ao fato de que havia mais cargas que estavam melhores ajustadas às características de área e peso dos navios e isso permitiu encontrar uma solução mais rapidamente. Formaram-se quatro rotas de duas plataformas.

As instâncias 1242T $\mathrm{T}_{1} \mathrm{C}, 1242 \mathrm{~T}_{2} \mathrm{C}, 1552 \mathrm{~T}_{1} \_\mathrm{C}$, $1552 \mathrm{~T}_{2} \mathrm{C}$ foram criadas com o intuito de verificar o comportamento do modelo para instâncias de maior escala em termos de quantidade de plataformas, cargas e navios. Neste caso, as dimensões e pesos das cargas foram propositalmente aumentados para forçar o uso de mais navios e assim avaliar como o modelo reagiria à inclusão de mais navios na solução. A solução das instâncias $1242 \mathrm{~T}_{1} \mathrm{C}_{\mathrm{C}} \mathrm{e}$ $1242 \mathrm{~T}_{2}$ C , que contêm 12 plataformas e se diferenciam pela quantidade de cargas demandas, utilizou os quatro navios disponíveis e gerou quatro rotas com no máximo três plataformas. Já na solução das instâncias $1552 \mathrm{~T}_{1} \_\mathrm{C}$ e $1552 \mathrm{~T}_{2} \mathrm{C}$, que apresentam 15 plataformas e também se distinguem pela quantidade de cargas, foram formadas cinco rotas de três plataformas usando cinco navios.

No que diz respeito aos gaps, com exceção da 933R 1 _C e da $1242 T_{1} \_$C, nota-se que as instâncias da Bacia de Campos, mesmo as que continham apenas quatro plataformas, não atingiram a solução ótima. Atribui-se como principal motivo de ocorrência deste gap o aspecto de carregamento do problema, influenciado por três fatores: 1) a quantidade de cargas arrumadas em um mesmo navio; 2) a quantidade de cargas demandadas por plataforma; e 3) a dimensão das cargas.

Em relação ao primeiro fator, observou-se que foi possível obter solução ótima arrumando até 13 cargas em um mesmo navio, conforme instâncias $733 R_{2} \_$E e 833R 1 _E. Em contrapartida, ao se analisar as instâncias $432 \mathrm{R}_{1 \_} \mathrm{C}$,
432R $\mathrm{R}_{2} \mathrm{C}, 532 \mathrm{R}_{1} \_\mathrm{C}, 533 \mathrm{R}_{1} \_\mathrm{C}, 632 \mathrm{R}_{1}$ C nota-se que apresentaram gap, mesmo contemplando menos plataformas que as instâncias $733 \mathrm{R}_{2}$ E e $833 \mathrm{R}_{1} \_\mathrm{E}$, pois efetuaram a arrumação de 15 a 30 cargas em um mesmo navio. A Figura 4a ilustra a solução gerada pelo modelo para a instância

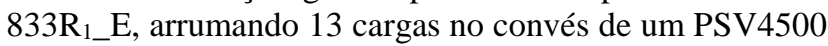
e a Figura $4 b$ mostra a solução para a instância $432 R_{2} \_$em um PSV3000, contendo 30 cargas.

No que concerne ao segundo fator, em geral, quanto maior a quantidade de cargas demandas por plataforma, mais difícil solucionar o problema e, portanto, mais complexo encontrar a solução ótima. A partir dos testes efetuados sabe-se que o modelo não suporta uma quantidade muito grande de cargas demandadas por plataformas. As

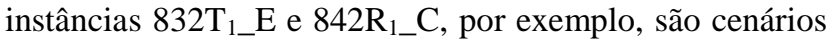
diferentes, mas apresentam a mesma quantidade de plataformas e de navios utilizados. Enquanto a demanda da instância $832 \mathrm{~T}_{1} \_$E variou até no máximo duas cargas por plataforma, a demanda da instância $842 \mathrm{R}_{1} \_\mathrm{C}$ variou até no máximo oito cargas por plataforma. Isso impactou na solução final, pois se verificou um gap de $0,00 \%$ para a primeira e de $43,14 \%$ para a segunda. Situação análoga pode ser percebida entre os pares $1242 \mathrm{~T}_{1 \_C} \mathrm{C} 1242 \mathrm{~T}_{2} \_\mathrm{C}$, e $1552 \mathrm{~T}_{1 \_} \mathrm{C}$ e $1552 \mathrm{~T}_{2} \mathrm{C}$.

No que tange ao terceiro fator, de modo geral, quanto menor a dimensão das cargas demandadas, o problema torna-se mais complexo, pois haverá uma maior dificuldade combinatorial. Por sua vez, o contrário também é verdadeiro, ou seja, quanto maiores as dimensões das cargas, mais fácil será encontrar a solução. Um exemplo desse caso pode ser observado na instância $842 \mathrm{~T}_{1} \_$E, que obteve gap de $0,00 \%$ e tempo de execução de dois segundos.

Os três fatores, embora apresentados separadamente, não se excluem. Ou seja, a ocorrência de um não implica na não ocorrência do outro. Por fim, pode-se afirmar que o aspecto de carregamento impactou fortemente na solução do problema e no tempo de execução do modelo.

Além disso, percebeu-se que a inserção de plataformas no problema exerce uma influência menor na questão do gap. É possível avaliar tal situação com base nas instâncias 933R 1 _C, 1242T 1_C $_{1}$ 1552T 1 _C. A instância 933R 1 _C é uma instância baseada em dados reais, em que cada plataforma demandou apenas uma carga, e obteve solução ótima. As instâncias $1242 \mathrm{~T}_{1} \_\mathrm{C}$ e $1552 \mathrm{~T}_{1} \_\mathrm{C}$, por sua vez, são de teste e a demanda por plataforma foi de duas cargas nos dois casos e para as duas instâncias foram arrumadas seis cargas em cada navio. Ou seja, a questão do carregamento não é preponderante nesta análise. Assim sendo, o gap de 0,01\% observado na instância $1552 \mathrm{~T}_{1} \_\mathrm{C}$ provavelmente ocorreu em função do aumento do número de plataformas de 12 para 15, já que a instância $1242 \mathrm{~T}_{1} \_\mathrm{C}$ apresentou gap de $0,00 \%$.

De modo geral, observou-se que instâncias com menor quantidade de plataformas se comportam melhor com o aumento do número de cargas demandadas por plataforma. Isso pode ser exemplificado pelas instâncias $432 \mathrm{R}_{2} \mathrm{C}$ e $842 \mathrm{R}_{1} \mathrm{C}$, que apresentavam demandas de até oito cargas por plataforma. Contudo, enquanto a primeira retornou um gap de $0,30 \%$, a segunda apresentou um gap de $43,14 \%$.

No que se refere ao equilíbrio náutico dos navios, analisando todo o conjunto de instâncias criadas, os momentos encontrados para cada navio foram, em sua maioria, 

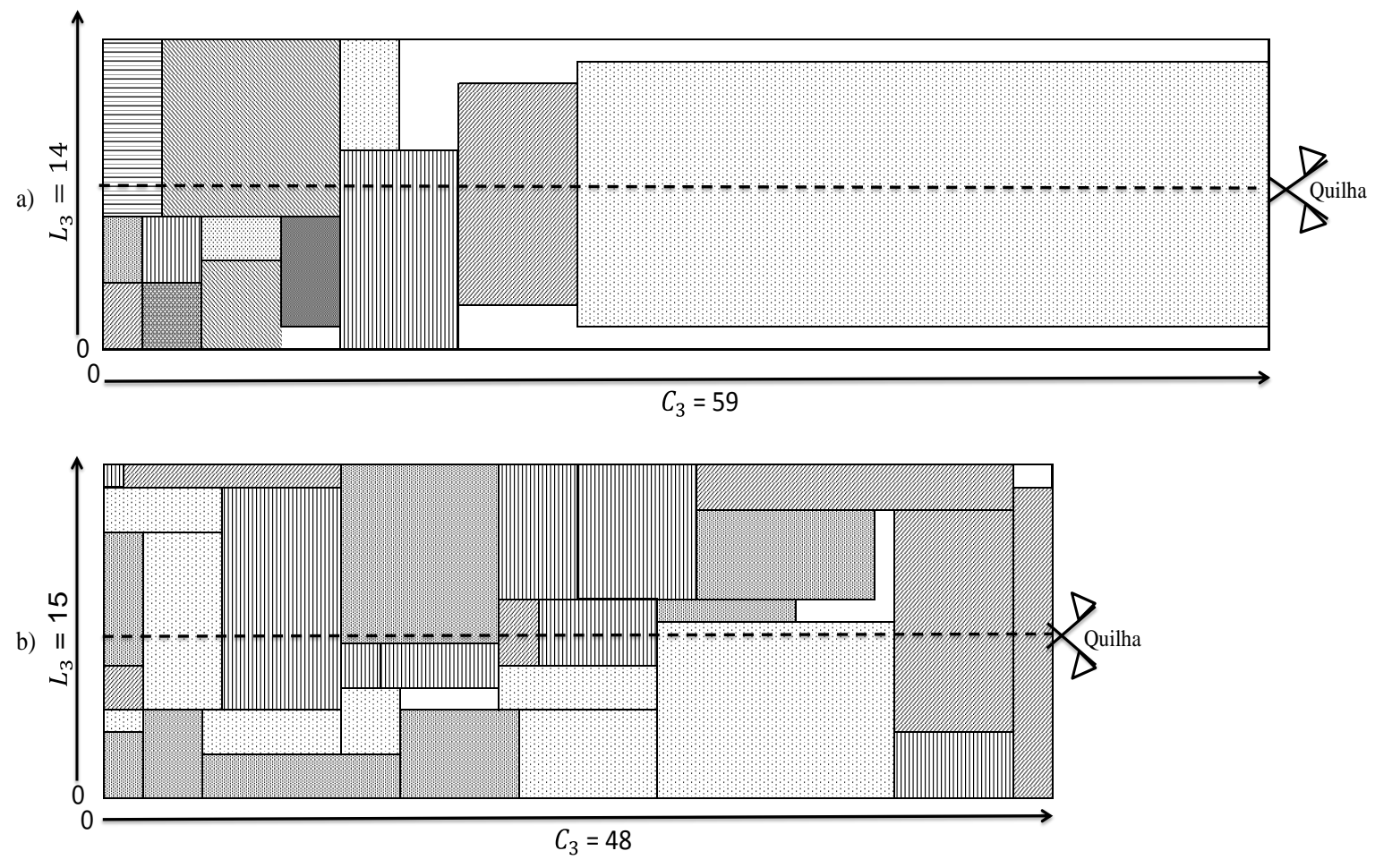

Figura 4. a) Arrumação obtida para a instância $833 R_{1}$ E em um PSV4500;

b) Arrumação obtida para a instância $432 R_{2}$ C em um PSV3000

menores que $1 \mathrm{t} . \mathrm{m}$, havendo dois casos (dois navios nas instâncias $1242 \mathrm{~T}_{1}$ C, $1552 \mathrm{~T}_{1} \_\mathrm{C}$ ) em que os navios atingiram momento igual a $1,485 \mathrm{t}$.m. Isso pode ser explicado pelo fato de que nessas instâncias, o peso atribuído à cada carga foi muito superior ao que que ocorre na realidade, pois havia necessidade de se testar o uso de mais de dois navios na construção das rotas. Em contrapartida, ao se avaliar apenas as instâncias baseadas em dados reais, observou-se, justamente pelo fato de as instâncias terem pesos mais próximos do real, o retorno de resultados mais eficazes, com o momento para todos os navios não sendo superior a 0,2 t.m. Isso demonstra que o modelo é eficaz no que se refere ao alcance do equilíbrio náutico do navio.

Por questões de sigilo da empresa em estudo, não foram disponibilizadas informações que permitissem comparações entre o modelo e a situação real quanto à disposição das cargas no convés e sobre o equilíbrio náutico.

\section{CONCLUSÃO}

Este artigo propôs um modelo PLIM para o 2LCVRP aplicado ao planejamento da logística de suprimento de plataformas offshore. O modelo proposto se diferencia por considerar uma frota heterogênea de navios e utilizar uma função objetivo que visa minimizar o número de navios, a distância percorrida, e a diferença entre os pesos distribuídos entre os bordos do navio com o intuito de manter o equilíbrio náutico.

O modelo foi testado em instâncias baseadas em dados reais da Petrobras no solver CPLEX 12.6, conseguindo obter economias em relação à distância percorrida de até $25 \%$. Contudo, a partir de testes efetuados também se observou a ocorrência de gaps, mesmo para instâncias contendo apenas quatro plataformas. A principal razão para isso foi atribuída ao aspecto de carregamento do problema, regido por três fatores: a quantidade de cargas arrumadas em um mesmo navio; a quantidade de cargas demandadas por plataforma; e a dimensão das cargas. Notou-se que o modelo não conseguiu revolver otimamente instâncias em que eram arrumadas mais de 13 cargas em um mesmo navio. Além disso, mesmo impactando menos na questão do gap, também há o aspecto de roteirização do problema, cuja dificuldade é expressa pelo aumento da quantidade de plataformas. Conseguiu-se resolver otimamente instâncias com até 12 plataformas. Como as características de roteirização e carregamento acabam se influenciando, de modo geral, observou-se que instâncias com menor quantidade de plataformas se comportaram melhor com o aumento do número de cargas demandadas por plataforma.

Sabe-se que a aplicação real demonstrada nesse artigo lidou com exemplos de pequena escala e que podem existir situações com mais cargas e mais plataformas, o que certamente dificultaria encontrar uma solução via abordagem exata, dada a dificuldade combinatorial inerente ao problema. Por isso, para alcançar resultados de grande escala é necessário desenvolver heurísticas e/ou meta-heurísticas.

Outra sugestão é a incorporação de outras variantes do 2L-CVRP ao problema, como a realização de operações de entrega e coleta de carga nas plataformas.

\section{AGRADECIMENTOS}

Os autores agradecem à FAPES (458/2013), ao CNPq (477357/2013-0) e à CAPES pelo apoio financeiro e à Petrobras pelo fornecimento dos dados do problema. 
Planejamento da logística de suprimento de plataformas Offshore por meio de um modelo matemático $2 \mathrm{~L}-\mathrm{CVRP}$ com frota heterogênea e equilíbrio náutico

\section{REFERÊNCIAS}

Aas, B.; Gribkovskaia, I.; Halskau Sr, Ø.; Shlopak, A. (2007) Routing of supply vessels to petroleum installations. Intern. Journal of Physical Distribution \& Logistics Management, v. 37, n. 2, p.164-179.

DOI: http://dx.doi.org/10.1108/09600030710734866.

Abdal-Hammed, M.K.; Hifi, M.; Wu; L. (2014) Large neighborhood search for the vehicle routing problem with twodimensional loading constraints. In: International Conference on Control, Decision and Information Technologies (CoDIT). IEEE, p. 054-059. DOI: 10.1109/CoDIT.2014.6996867.

A Gazeta. Novos terminais e plataformas no Estado: Plano confirma nova unidade na área de gás no Norte até 2016. Disponível em:

gazetaonline.globo.com/_conteudo/2012/06/noticias/a_gazeta/ec onomia/1287494-novos-terminais-e-plataformas-no-estado.html. Acesso em 15 de janeiro de 2014

Almeida, M. R. de. (2009) Algoritmos Genéticos Aplicados a Programação de Embarcações de Apoio às Operações "Offshore". Anais do XLI Simpósio Brasileiro de Pesquisa Operacional, SOBRAPO, Bahia.

Araujo, R. R. de. (2010) Uma abordagem de resolução integrada para os problemas de roteirização e carregamento de veículos. $173 \mathrm{f}$. Tese Doutorado, PPGEP, UFRGS, Porto Alegre.

Bin, W.; Hong, C.; Zhi-Yong, C. (2013) Artificial bee colony algorithm for two-dimensional loading capacitated vehicle routing problem. In: ICMSE, IEEE, p. 406-412. DOI: 10.1109/ICMSE.2013.6586313.

Brejon, S.; Brinati, M. A. (1998) Algoritmo para resolução do problema de programação do transporte de suprimentos para unidades marítimas de exploração de petróleo. Dissertação de Mestrado. Escola Politécnica, Universidade de São Paulo.

Click Macaé. Diagrama do Fluxo de Petróleo na Bacia de Campos. Disponível em:

www.clickmacae.com.br/?sec=361\&pag=pagina $\& \operatorname{cod}=545$. Acesso em 10 de janeiro de 2015.

Côté, J.-F.; Gendreau, M.; Potvin, J.-Y. (2013) The Vehicle Routing Problem with Stochastic Two-Dimensional Items, CIRRELT-2013-84.

Dominguez, O.; Juan, A. A.; Faulin, J. A. (2014a) A biasedrandomized algorithm for the two-dimensional vehicle routing problem with and without item rotations. Intern. Transactions in Operational Research, p. 1-14. DOI:10.1111/itor.12070.

Dominguez, O.; Juan, A. A.; Barrios, B.; Faulin, J.; Agustin, A. (2014b) Using biased randomization for solving the twodimensional loading vehicle routing problem with heterogeneous fleet. Annals of Operations Research, p. 1-22.

DOI: $10.1007 / \mathrm{s} 10479-014-1551-4$.

Duhamel, C.; Lacomme, P.; Quilliot, A.; Toussaint, H. (2011) A multi-start evolutionary local search for the two-dimensional loading capacitated vehicle routing problem. Computers \& Operations Research, v. 38, n. 3, p. 617-640.

DOI: $10.1016 /$ j.cor.2010.08.017.

Fagerholt, K.; Lindstad, H. (2000) Optimal policies for maintaining a supply service in the Norwegian Sea. Omega, v. 28, n. 3, p. 269-275. DOI: 10.1016/S0305-0483(99)00054-7.

TRANSPORTES v. 23, n. 4 (2015), p. 67-78
Ferro, F.; Teixeira, P. (2009) Os desafios do Pré-Sal. Brasília: Câmara dos Deputados. 78 p.

Fuellerer, G.; Doerner, K. F.; Hartl, R. F.; Iori, M. (2009) Ant colony optimization for the two-dimensional loading vehicle routing problem. Computers \& Operations Research, v. 36, p. 655 - 673. DOI: 10.1016/j.cor.2007.10.021.

Friedberg, D. O.; Uglane, V. T. (2013) Routing and Scheduling of Platform Supply Vessels: Case from the Brazilian Petroleum Industry. 2013. Thesis (Master in Applied Economics and Optimization) - Department of Industrial Economics and Technology Management, Norwegian University of Science and Technology (NTNU), Norway.

Gendreau, M.; Iori, M.; Laporte, G.; Martello, S. (2008) A Tabu Search heuristic for the vehicle routing problem with twodimensional loading constraints. Networks, v. 51, n. 1, p. 4-18. DOI: 10.1002/net.20192.

Gribkovskaia, I.; Laporte, G.; Shlopak, A. (2008) A tabu search heuristic for a routing problem arising in servicing of offshore oil and gas platforms. Journal of the Operational Research Society, v. 59, n. 11, p. 1449-1459. DOI: 10.1057/palgrave.jors.2602469.

Halvorsen-Weare, E. E.; Fagerholt, K. (2011) Robust supply vessel planning. Network Optimization. Springer Berlin Heidelberg, p. 559-573. DOI: 10.1007/978-3-642-21527-8_62.

Halvorsen-Weare, E. E.; Fagerholt, K.; Nonås, L. M.; Asbjørnslett, B. E.(2012) Optimal fleet composition and periodic routing of offshore supply vessels. European Journal of Operational Research, v. 223, p. 508-517.

DOI : 10.1016/j.ejor.2012.06.017.

Hamdi-dhaoui, K. ; Labadie, N.; Yalaoui, A. (2012) Problème de tournées de véhicules avec chargement bidimensionnel et contraintes de conflits partiels multi-objectif. Proceedings of MOSIM'12.

Henty, F. C.; Meza, B. M.; Vianna, D. S.; Vianna, M. De F. D. (2012) Um Modelo Matemático para a Programação e Roteirização de Embarcações de Apoio à Exploração de Petróleo Offshore. In: Encontro Nacional de Engenharia de Produção, 32, 2012, Bento Gonçalves (RS). Anais... Bento Gonçalves (RS): ABEPRO.

Iori, M.; Gonzáles, J. J. S.; Vigo, D. (2007) An exact approach for the vehicle routing problem with two-dimensional loading constraints. Transportation Science, v. 41 (2), p. 253-264. DOI: http://pubsonline.informs.org/doi/abs/10.1287/trsc.1060.0165.

Khebbache-hadji, S. ; Prins, C.; Yalaoui, A.; Reghioui, M. (2013) Heuristics and memetic algorithm for the twodimensional loading capacitated vehicle routing problem with time windows. Central European Journal of Operations Research, v. 21, n. 2, p. 307-336. DOI: $10.1007 / \mathrm{s} 10100-011-0204-9$.

Leung, S. C. H.; Zheng, J.; Zhang, D.; Zhou, X.(2010) Simulated annealing for the vehicle routing problem with twodimensional loading constraints. Flexible services and manufacturing journal, v. 22, p. 61-82. DOI: $10.1007 / \mathrm{s} 10696-010-9061-4$.

Leung, S. C. H.; Zhou, X.; Zhang, D.; Zheng, J. (2011) Extended guided tabu search and a new packing algorithm for the two-dimensional loading vehicle routing problem. Computers \& 
Operations Research, v. 38, p. $205-215$.

DOI: 10.1016/j.cor.2010.04.013.

Leung, S. C. H.; Zhang, Z..; Zhang, D.; Hua, X.; Lim, M. K. (2013) A meta-heuristic algorithm for heterogeneous fleet vehicle routing problems with two-dimensional loading constraints. European Journal of Operational Research, v. 225, p. 199-210. DOI: 10.1016/j.ejor.2012.09.023.

Lopes, P. H. M. (2011) Uma Solução para o Problema de Roteamento de Embarcações de Apoio "Offshore" através da Metaheurística RTR. Dissertação de Mestrado, COPPE, UFRJ, Rio de Janeiro.

Martínez, L.; Amaya, C. A. (2013) A vehicle routing problem with multi-trips and time windows for circular items. Journal of the Operational Research Society, v. 64, n. 11, p. 1630-1643. DOI: $10.1057 /$ jors.2012.128.

Muñoz, A. L. (2011) Solución al problema de ruteo de vehículos con restricciones de capacidad y reordenamiento de carga en los sitios de demanda. $84 \mathrm{f}$. Tesis Doutorado, PUC de Chile, Chile.

Norlund, E. K.; Gribkovskaia, I. (2013) Reducing emissions through speed optimization in supply vessel operations. Transportation Research Part D: Transport and Environment, v. 23, p. 105-113. DOI: 10.1016/j.trd.2013.04.007.

Panamarenka, K. Minimization of emissions in periodic supply vessel planning through speed optimization. 2011. Thesis (Master in Logistics) - Molde University College, Norway, 2011.

Ribeiro, L. S.; Iachan, R. (2009) Dimensionamento da Frota de Supridores para Plataformas de Petróleo. Anais do XLI Simpósio Brasileiro de Pesquisa Operacional, SOBRAPO, Bahia.

Shen, Y.; Murata, T. (2012) Pick-up Scheduling of Twodimensional Loading in Vehicle Routing Problem by using GA. In: Proceedings of the International MultiConference of Engineers and Computer Scientists, IMECS, v. 2, p. 1532-1537, Hong Kong.

Shyshou, A.; Fagerholt, K.; Gribkovskaia, I.; Laporte, G. (2012) A large neighbourhood search heuristic for a periodic supply vessel planning problem arising in offshore oil and gas operations. INFOR: Information Systems and Operational Research, v. 50, n. 4, p. 195-204.

DOI: http://dx.doi.org/10.3138/infor.50.4.195.

Sopot, E.; Gribkovskaia, I. (2014) Routing of Supply Vessels to with Deliveries and Pickups of Multiple Commodities. Procedia Computer Science, v. 31, p. 910-917.

DOI: $10.1016 /$ j.procs.2014.05.343.

Strodl, J.; Doerner, K.F.; Tricoire, F.; Hartl, R.F. (2010) On index structures in hybrid metaheuristics for routing problems with hard feasibility checks: An application to the 2-dimensional loading vehicle routing problem. In: Blesa, M.J.; Blum, C.; Raidl, G.; Roli, A.; Sampels, M. Hybrid Metaheuristics, v. 6373 of Lecture Notes in Computer Science, p. 160-173, Springer Berlin Heidelberg. DOI: 10.1007/978-3-642-16054-7_12.

Vaquero, T. S.; Costa, G.; Tonidandel, F.; Igreja, H.; Silva, J. R.; Beck, C. (2012) Planning and Scheduling Ship Operations on Petroleum Ports and Platforms. In: Proceedings of the Scheduling and Planning Applications Workshop. p. 8-16.
Vianna, D. S.; Meza, E. B. M.; Hentzy, F. C.; Martins, C. B.; Medeiros, A. P. (2012) Heurísticas Baseadas em Busca Local para a Programação e Roteirização de Embarcações de Apoio à Exploração de Petróleo OffShore, Analisando Múltiplas Estruturas de Vizinhança. Anais do XLIV SOBRAPO, Rio de janeiro.

Zachariadis, E. E.; Kiranoudis, C. T.; Tarantilis, C. D. (2009) A Guided Tabu Search for the Vehicle Routing Problem with twodimensional loading constraints. European Journal of Operational Research, v. 195, n. 3, p. 729-743. DOI: 10.1016/j.ejor.2007.05.058.

Zachariadis, E. E.; Tarantilis, C. D.; Kiranoudis, C. T. (2013) Integrated distribution and loading planning via a compact metaheuristic algorithm. European Journal of Operational Research, v. 228, p. 56-71. DOI: 10.1016/j.ejor.2013.01.040.

Wei, L., Zhang, Z., Zhang, D., Lim, A. (2015) A variable neighborhood search for the capacitated vehicle routing problem with two-dimensional loading constraints. European Journal of Operational Research, v. 243, n.3, p. 798-814.

DOI: 10.1016/j.ejor.2014.12.048. 